Journal of Computer Science 2(8): 607-611, 2006

ISSN 1549-3636

(c) 2006 Science Publications

\title{
Microcontroller Based Power Supply
}

\author{
${ }^{1}$ Tanvir Singh Mundra, ${ }^{2}$ Er. Satwinder Singh Sachdeva and ${ }^{2}$ K.S. Kahlon \\ M/S Pulse Engineering, D-153, Industrial Area Phase-8, SAS Nagar Mohali, Punjab, India \\ Department Computer Science Engineering, Rayat Institute of Engineering and IT, Rail Majra \\ Department Computer Science Engineering, Guru Nank dev University Amritsar
}

\begin{abstract}
These days, majority of electronic devices work on DC power source, so there was a requirement of a reliable and customized power supply. Generally, the requirements are not too varied, but still they require every time a new hardware designing. The idea presented here is to build a microcontroller controlled power supply ${ }^{[1]}$ that is flexible enough to meet different customer requirements, with minor software changes and no corresponding hardware change. Hardware issues are discussed, with a goal of developing a generalized power supply that has programmable output voltage and current. It is able to recognize faults and take corrective actions to prevent any permanent damage to the system. The system discussed here is capable of functioning independently by its own without any intervention from the user. The system finds application at remote sites to automatically manage primary (AC) and secondary (Battery) power sources to provide smooth uninterrupted power output even during switchovers between AC and DC power sources. It would also be helpful to insurance companies who expect that the products covered by them be reliable, robust and not prone to be damages.
\end{abstract}

Key words: Real time system, embedded system design, power supply diagnostic firmware

\section{INTRODUCTION}

The trend and demand these days are to develop systems that are reliable and intelligent. People need products that are reliable and do not require constant supervision $^{[2]}$. From the manufacturer point of view, they require a product that is highly reliable and does not damage if the parameters run out of specifications or even on mishandling by the user. To increase the market base of their products the manufacturer tries to put enough intelligence in the system so that it is capable of functioning by itself. Embedded system's is one such area, which fits well in these situations.

The microcontroller based power SUPPLY is developed with a view to make a flexible and an intelligent power supply controlled by a microcontroller of 8051 family $^{[3]}$ and to eliminate the drawbacks or limitations of the existing systems. They are discussed.

Most of the existing systems are hardwired and expensive, which increases the production and procurement cost. The PCBs are larger and complex because of low component integration ${ }^{[4-6]}$.

Due to hardwired logic the flexibility is low and a particular PCB circumvents to the specified requirements only. There is no room for change, as any modification in specification requires redesigning. The system parameters such as battery charging voltage, current are not programmable.

They do not have the ability to generate logs or have periodic automatic self-testing techniques. User interface is not that interactive or informative.
Description: The design of the microcontroller based power supply circuit satisfies the following requirements:

Read the voltages at 8 given points.

* Indicate the battery or mains related errors on LCD or LED's and operate relevant relays.

* Display the following parameter values - Error messages, Battery voltage, Battery charging current, Output voltage, Load current, Battery temperature, System temperature, Current time and date.

* Logging of upto 250 errors along with time and date of its occurrence in non-volatile EEPROM.

* Editor to update certain parameters to enable the user meet his custom requirements. These are Temperature at which to switch on the fan, Maximum Load Current and Maximum Battery Charging Current.

* Automatic testing of battery every $12 \mathrm{hrs}$.

The project is implemented in two stages:

Hardware design: This required the designing of the microcontroller circuit along with the other hardware as ADC, LCD, RTC ${ }^{[7]}$ and keyboard design ${ }^{[8]}$ (Fig. 1). At the center of the whole system is the microcontroller 89LS8252 ${ }^{[9]}$. To enable it to read the analog signals an Analog to Digital converter ADC $0808^{[10]}$ is used. Necessary amplification is provided before the ADC. 


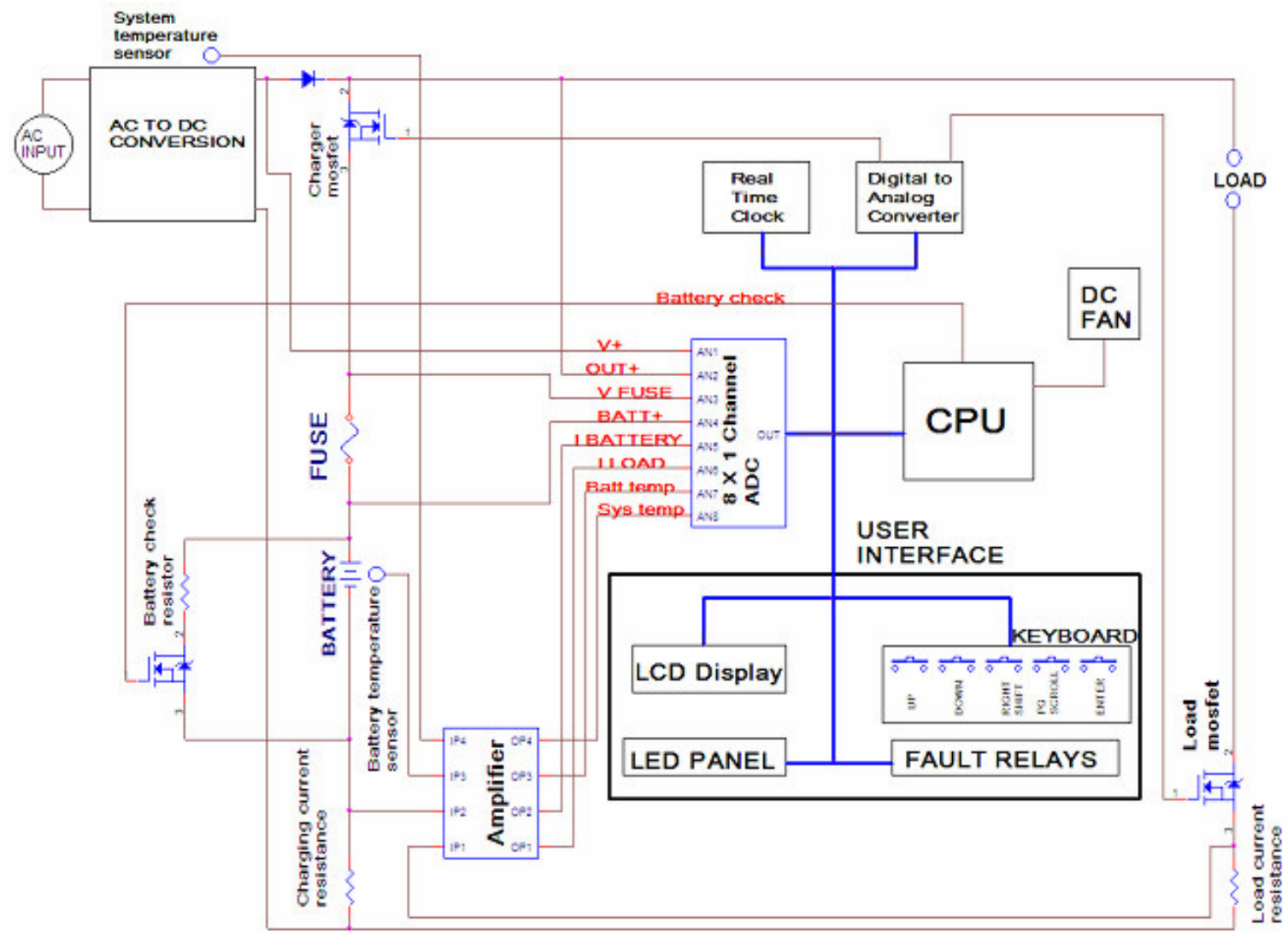

Fig. 1: Block diagram

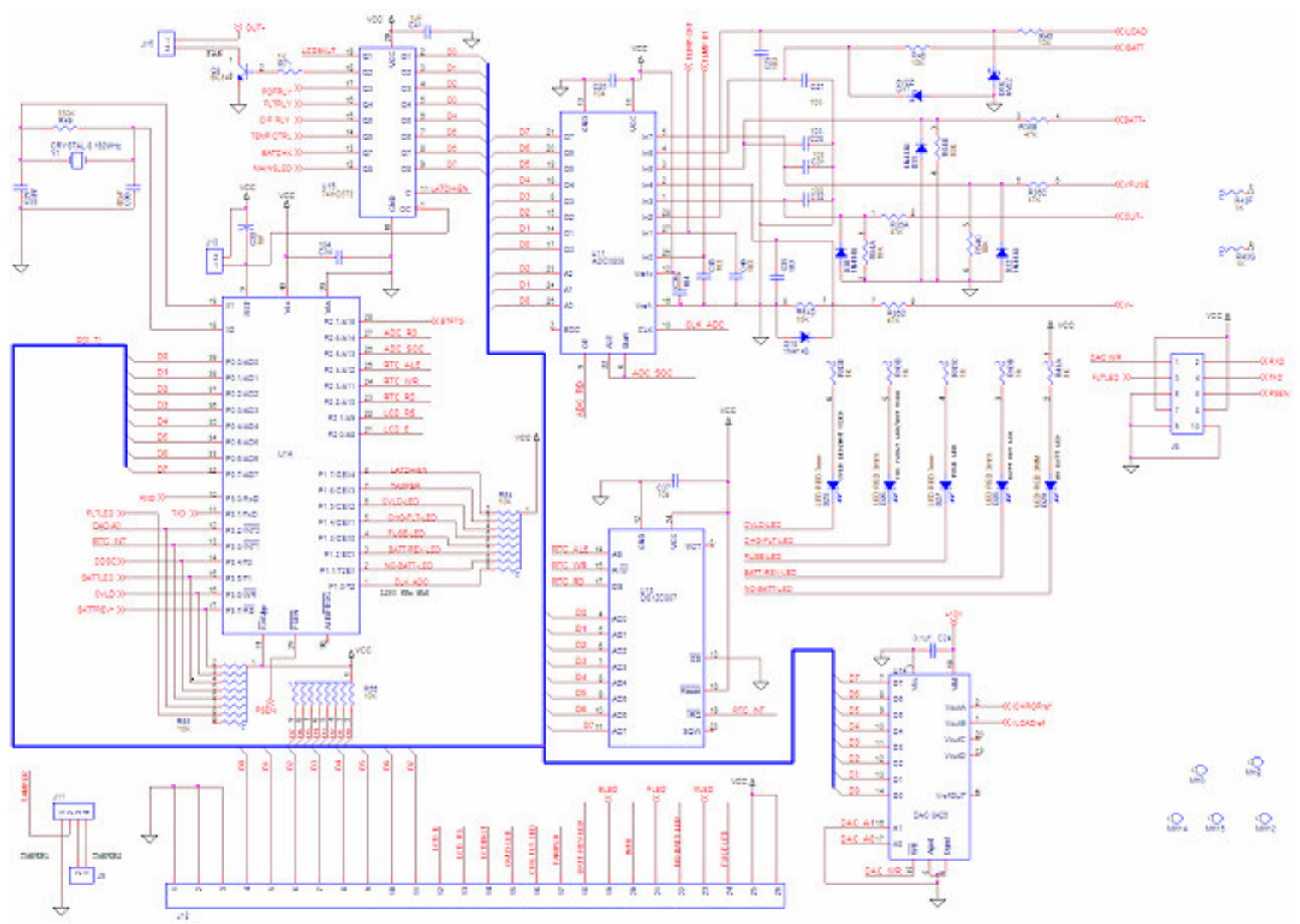

Fig. 2: Circuit diagram processor board 


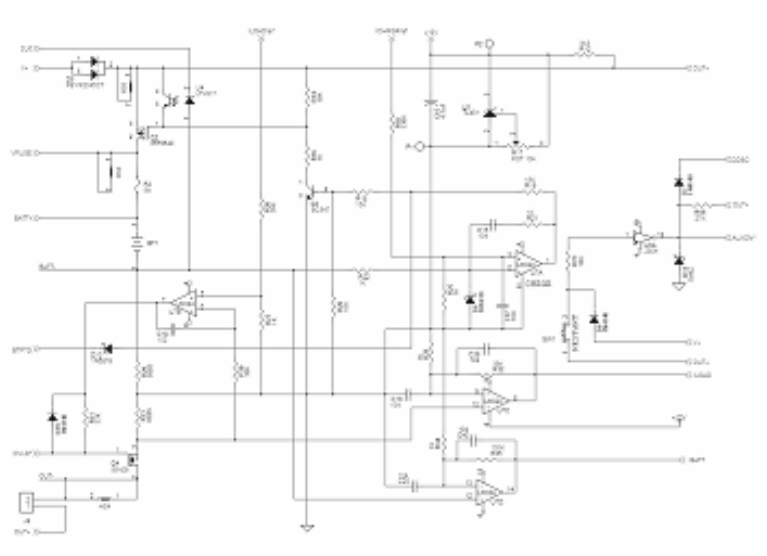

Fig. 3: Circuit diagram interface board

To control voltages a 4 channel DAC $8426^{[11]}$ has been used.

The hardware designed is flexible and can be easily scaled to the requirements (Fig. 2). The microcontroller (89LS8252) used has an internal $2 \mathrm{~K}$ byte EEPROM, 256 bytes RAM and $8 \mathrm{~K}$-code memory that is adequate to store program data, parameters and logs.

Keyboard is interrupt driven so the controller need not waste time in scanning keys. Whenever a key is pressed, an interrupt is received and the controller can read the pins to which each key is attached. It has a 16 $\mathrm{X} 2$ character display interface with backlight for user interaction and to display system's health and parameters. Each individual pin of the controller can be programmed as input only, output only or made bidirectional eliminating the need of external digital $\mathrm{read} /$ write ports for control and monitoring. There are 5 on board LEDs, 3 off board LEDs and 3 relays that can be used to indicate various error conditions.

To measure certain analog signals that are in the range of 0-28 volts they are linearly scaled down. Other voltages as developed across the current resistances are in the range of mill volts; they are accurately amplified to be in a range of 0-5 volts, this is required so that they can be measured by the ADC. Further, each signal requires filtering to remove high frequency transients before feeding them to the ADC inputs for accurate measurement. The controller generates the clock to the $\mathrm{ADC}$ and this is software programmable. As such, a balance between speed and accuracy of ADC conversion can be achieved ${ }^{[12]}$.

The Real Time Clock has an internal battery backup, which enables it to maintain accurate time even if the system is switched off; which is a necessary requirement to maintain accurate error logs.

As the response time of the system is 250 mses in the worst case, some additional hardware protection, an optocoupler has been used to shut down the battery charging mosfet when the battery is reverse connected (Fig. 3). The load mosfet is also controlled by a comparator which does not allow the load current to exceed the programmed limit in case of a short circuit until the controller recognizes the overload condition and shuts off the load.

Temperature sensor's (LM335) are used to measure system and battery temperature. It is a constant voltage source that gives an output of $1 \mathrm{mV} /{ }^{\circ} \mathrm{K}$. The output is amplified to make it operate from $0^{\circ} \mathrm{C}$ to $50^{\circ} \mathrm{C}$. The controller can measure the temperature with $0.2^{\circ} \mathrm{C}$ accuracy. The system temperature is monitored to switch on the fan if the gets heated up.

The charging current of the battery is temperature dependent and charging is done in two steps, namely boost charge followed by trickle charge. The system continuously monitors the charging status of the battery and switches off the charger as soon as the battery gets charged to prevent overcharging. All this enhances battery life and performance.

Software Design: The software is written in C based KEIL COMPILER. The compiler converts the code written in C language into its equivalent 8051 machine code. The compiler also supports other microcontrollers of the 8051 family. Using this compiler the whole code has been written under different header files for easy understanding and enhanced clarity.

At power up it is the task of the software to program each controller pin and to initialize hardware devices. The RTC $12 \mathrm{C} 887^{[13]}$ is programmed to generate hardware interrupt every $250 \mathrm{~ms}$, the service routine and control code is executed once. This limits the maximum response time of the system is $250 \mathrm{~ms}$. The controller has an in built watchdog protection which is programmed to reset the system in a maximum of $512 \mathrm{~ms}$, this restricts the system down time to $512 \mathrm{~ms}$.

At every interrupt of $250 \mathrm{~ms}$, the system looks for any errors and takes appropriate actions to enable safe and proper system functioning. The error messages displayed on the LCD are updated every $250 \mathrm{~ms}$. System parameters as voltages, currents and temperatures are refreshed after $1 \mathrm{sec}$ and not $250 \mathrm{~ms}$, so that the least significant digits does not change too frequently. It also displays the current time and date on the LCD. To brief the user about the various errors or emergencies there are upto 8 LEDs and 3 relays whose status is updated and each corresponds to different error conditions.

The software is responsible for updating the DAC output voltage so that battery charging current can be controlled according to the battery temperature. The DAC value for each temperature is stored in a look up table in the non volatile code memory. Similarly, the value of the maximum load current entered by the user can be controlled by the other DAC's output.

Each time an error is generated it is logged in the non-volatile memory EEPROM along with the time, date and nature of fault. The user can see these logs, 
even after power failure or system shut off. There can be upto $250 \operatorname{logs}$ and the latest $\log$ is at $1^{\text {st }}$ index. If the logs are full, the new log entry is overwritten over the last entry in the in FIFO order. The EEPROM is also used to store various user programmable parameter values. The EEPROM write has been disabled through software after each write so that it is not corrupted accidentally at power up.

There are separate pages through which the user can set various parameters so that the operation of the system could be easily customized according to varied user requirements. There are ranges assigned to each of these updateable parameters and a responsibility of the software to ensure that the user does not enter out of bound values, accidentally or intentionally and harm the system.

The battery charging mosfet is switched on for 8 seconds, then the charger is switched off for $250 \mathrm{~ms}$ and during this time battery voltage is read, the charger and battery fuse is checked. In case the battery voltage is found below some threshold value then the controller automatically shuts down the system. After every 12 hrs and if the system is running on mains, then the battery is put to load of $33 \Omega$ for 10 seconds and after that if the battery voltage is greater (Less) than threshold it is logged as good (Suspect). This test can also be initiated by the operator. One thing the software has to ensure is that if the mains go off then the battery testing $33 \Omega$ load has to be immediately removed as the output load is also transferred to the battery and it may burn the battery or heat it up.

The software multiplexes between - Calculation of $\mathrm{ADC}$ values to corresponding Voltages and Ampere, User commands, Keyboard reads, Updation of LED and relay status, Logging of errors, Display refresh at $250 \mathrm{msec}$ and Execution of control program.

Alarms: Various alarms are discussed below:

OVER LOAD: It happens when load current exceeds the set limit. This is detected by measuring the ILOAD value of the ADC. The processor sends a 3sec OVLD signal to forcibly shut the load mosfet and thus stopping the current flow in the load.

AC ON /AC OFF: If the input DC voltage is > or < than $25 \mathrm{~V}$. This can be detected by reading the $\mathrm{V}+$ value of ADC.

TAMPER: If the power supply is run with its case open. This is detected by the processor's read input TAMPER which is shorted to ground if the case is closed and is read as logic 1 when the due to the open case.

NO LOAD: If load current at the output is less than $0.2 \mathrm{~A}$. This can be detected by measuring the ILOAD value of the ADC.
BATT OK: If there are no battery related faults, namely from alarm no. 6 to 9 . This can be detected by checking the condition flags.

BAT REV: If the battery is reverse connected. This can be detected by reading the BATTREV* signal, which is made low by the optocoupler when the battery is reverse connected.

NO BATT: If the battery is not connected. This can be detected by first switching off the charger by making BTFTG signal low and then if the value at the BATT+ signal of $\mathrm{ADC}$ is less than $3 \mathrm{~V}$ it is recognized as no battery.

BAT LOW: If the battery voltage has fallen below 23 V. This can be detected by switching off the charger and if the voltage at BATT+ signal of ADC is less than $23 \mathrm{~V}$.

BT FUSE: If battery fuse has blown due to flow of excessive current through the fuse. This can be detected by switching of the charger and if the battery is present then the voltage at the VFUSE signal of ADC should not be $<$ than $3 \mathrm{~V}$.

CHG FLT: If the battery is not being charged. Switch off the charger if the battery voltage is below its maximum voltage and the mains is present and fuse is ok. If the value at ILOAD is less than $0.05 \mathrm{~A}$ the charger is termed as bad.

DDSC (Deep Discharge): The power supply is automatically switched OFF at a battery voltage less than $21.2 \mathrm{~V}$. This can be done when the AC is off and the battery voltage has fallen below $21.2 \mathrm{~V}$. The controller makes the DDSC signal HI and shuts down the voltage regulator.

Battery good/suspect: If the battery automatic test passes/fails. To do this the mains should be present. The processor sends a HI BATCHK signal, which switches on the battery check mosfet connecting 33E load across battery. The BATCHK signal is then made low and if the battery voltage is above cutoff it is termed as BATTERY GOOD else BATTERY SUSPECT.

\section{CONCLUSION}

It is a software controlled microcontroller based system, which makes it intelligent, independent of operator supervision and can adapt to a restricted set of changed requirements without any hardware change.

The system can log upto 250 faults and events with time and date of occurrence in a non-volatile memory. The manufacturer can verify from the logged events whenever the customer mishandles or fiddles with the 
power supply. This helps in resolving the issues related to providing guarantee or warrantee benefits.

It can handle most of the alarms by itself and adjust its parameters as the situation demands. Three relays provided indicate emergency fault conditions.

Due to fast response of the system, the controller is able to shut down the power supply even in case of short circuit at the output of the supply.

The charging of the battery is temperature dependent, this enhances the battery life and its performance. Battery is automatically checked every 12 hrs and the result logged.

Limitations: The system's reaction time is $250 \mathrm{~ms}$ so in some cases additional protective hardware is required.

* The system output voltage is not programmable.

* The system cannot locate own hardware faults.

* To ensure proper functioning of the system one has to make ensure that the DC voltage is kept greater than battery voltage, so that it can be charged properly.

Future scope: The future work can be done on the development of software that makes the system selfdiagnostic, detecting damage of components in itself. E.g., Some controllers are there which generate an interrupt if the output of a pin does not match the sent value.The separate controller portion can be designed so that it can be put on to any supply and after programming various parameters in the controller any power supply could be controlled.

\section{ACKNOWLEDGEMENT}

I would like to express my gratitude to Managing Director, Pulse Engineering in providing me facilities, designing of 89LS8252 based power supply PCB, support in purchase of components and timely guidance as and when I had technical problems, I would like to thank the staff for their support in providing me the help I required for procuring of components and PCB fabrication.

\section{REFERENCES}

1. Moksimovic, D., R. zane and R. Erickson, 2004. Impact of digital control in power electronics. IEEE Intl. Symp. on Power Semiconductor Devices and ICs. Kitakyushu, Japan, pp: 13-22.

2. Mammano, B. and L. Bahra, 2005. Safety considerations in power supply design. Unitrode (TI) Power Supply Design Seminar, SEM1600 2004/2005.

3. Kenneth, J.A., 1993. The 8051 Microcontroller: Architecture, Programming and Applications. St. Paul: West Publishing Company.

4. Abraham, I.P., 1998. Switching Power Supply Design. 2nd Edn. McGraw-Hill, New York.

5. Power Supply Design by Jerrold Foutz

6. Lloyd H. Dixon, Jr. Switching Power Supply Review.

7. Scott, I.McK., 1995. The 8051 Microcontroller. Prentice Hall. 2nd Edn.

8. Balogh, L., 2005. A practical introduction to digital power supply control. Unitrode (TI) Power Supply Design Seminar, SEM1600 2004/2005.

9. Atmel, datasheet AT 89LS8252.

10. National Semiconductor, datasheet ADC 0808 / ADC 0809.

11. Analog Devices, datasheet DAC 8426 (Norwood, U.S.A. Revision C)

12. R. Erickson and D. Moksimovic, "Fundamentals of Power Electronics", Second Edition, Kluwer Academic Publishers, 2000, ISBNO-7923-7270-0.

13. Dallas Semiconductor, datasheet DS 12 C 887 